\title{
CRECIMIENTO Y RENDIMIENTO DEL BANANO (Musa AAA) BAJO CICLOS CONSECUTIVOS DE ASPERSIÓN CON GLIFOSATO ${ }^{1}$
}

\author{
Renán Agüero ${ }^{2}$, Leonardo Pérez ${ }^{3}$, Mauricio Guzmán ${ }^{3}$
}

\begin{abstract}
RESUMEN
Crecimiento y rendimiento del banano (Musa AAA) bajo ciclos consecutivos de aspersión con glifosato. El herbicida Ranger (glifosato a $240 \mathrm{~g} / \mathrm{l}$ ) se usa en Costa Rica para el control de malezas en banano desde 1991. La presente investigación se llevó a cabo para determinar si el uso continuo de este herbicida en banano, asperjado como lo recomienda la compañía fabricante, tiene algún efecto sobre su crecimiento y desarrollo. El experimento se inició en noviembre de 1996 en la finca San Pablo, ubicada en la zona Atlántica de Costa Rica. En ese lugar se escogieron al azar unidades de producción en un estado fenológico similar (todas mostrando abuela-madre-hijo). Sobre dichas unidades se evaluaron varios tratamientos con glifosato desde una sóla aspersión, hasta ciclos consecutivos cada siete semanas. Se incluyó un testigo con deshierbas manuales cada mes. Los tratamientos evaluados no afectaron el diámetro del pseudotallo, altura de la planta, número de hojas, ancho de la hoja más joven, ni el peso de raíces en los hijos asperjados con el herbicida. Tampoco se afectó la sincronización en crecimiento entre madres e hijos, ni hubo diferencias entre tratamientos en producción y calidad de fruta.
\end{abstract}

\begin{abstract}
Effect of glyphosate on banana growth and yield. The objective of this study was to determine the effect of successive use of glyphosate - sprayed as recommended by the manufacturer - in banana growth and yield. The experiment started in November, 1996 at the San Pablo farm located in the Atlantic region of Costa Rica. Production units at a similar phenological stage (al1 of them showing grandmother-mother-son) were ramdomly chosen. Several treatments with glyophosate were analyzed on said units, ranging from one application to consecutive-application cycles every seven weeks. A control - hand-weeded once a month - was included. The treatments evaluated did not affect the diameter of pseudostems, plant height, number of leaves, width of youngest leaf, or root weight of sprayed sons. Likewise, growth synchronization between mothers and sons, as well as fruit yield and quality, were not affected.
\end{abstract}

\section{INTRODUCCIÓN}

Por muchos años el combate de malezas en plantaciones establecidas de banano se basó en el paraquat. Este herbicida se suele asperjar en ciclos mensuales. La rápida aparición de síntomas en las malezas asperjadas, así como un buen margen de selectividad al cultivo y su bajo precio, promovieron una rápida adopción de este herbicida por los productores. Por otra parte, la acumulación a largo plazo en el suelo y la alta toxicidad potencial a animales y al hombre son aspectos a considerar cuando se usa paraquat (World Health Organization, 1984).
El herbicida glifosato surgió como una opción para el control de malezas en banano. Este herbicida muestra acción sistémica que provee un control de amplio espectro (Franz et al., 1997). Lo anterior y la rápida biodegradación y des activación en el suelo, además de su baja toxicidad a fauna en general y al hombre, pennitieron un rápido crecimiento en las ventas de herbicidas basados en glifosato a partir de 1991. La presentación del glifosato a $240 \mathrm{~g} / \mathrm{l}$, conocida comercialmente como Ranger, es el producto dominante a la fecha.

El glifosato no muestra actividad herbicida en el suelo (Mensink y Janssen, 1994); sin embargo, el núme-

1 La empresa Monsanto financió la investigación independiente que se reporta en este documento.

2 Centro de Investigación en Protección de Cultivos (CIPROC), Universidad de Costa Rica, Facultad de Agronomía. Tel. (506) 207-4141; fax. (506) 234-6164. E-mail: raguero@ cariari.ucr.ac.cr

3 Corporación Bananera Nacional, Costa Rica. Tel. (506) 763-3176; fax (506) 763-3055. 
ro de aplicaciones por año que se ocupan con este herbicida es inferior comparado con paraquat. Se cree que lo anterior se debe aliento desarrollo de síntomas en plantas asperjadas con glifosato, con lo que, mientras mueren las malezas pueden funcionar como una cobertura viva temporal no competitiva, que inhibe el crecimiento de nuevas plántulas. Esto también podría ser útil para reducir erosión de suelos en contraste con otras estrategias de control de malezas que permiten la presencia de suelo desprovisto de vegetación durante más tiempo.

Para no dañar al cultivo, debe evitarse el contacto del glifosato con hojas de banano en activa fotosíntesis. En la mayoría de los casos, las hojas de hijos espada entran en fotosíntesis activa cuando estas plantas muestran más de un metro de altura. Se debe asperjar manteniendo la boquilla por debajo de los $75 \mathrm{~cm}$ de altura.

\section{Objetivos}

General: Corroborar si el uso del herbicida glifosato, con base en las recomendaciones de la fábrica Monsanto, es selectivo a unidades de producción de banano.

Específicos: 1- Determinar si aspersiones de glifosato sobre hijos espada dependientes de banano afectan su crecimiento o desarrollo. 2- Determinar si aspersiones de glifosato sobre hijos espada dependientes de banano, afectan el rendimiento y calidad de fruta de las madres o de esos hijos.

\section{MATERIALES Y MÉTODOS}

\section{Localización:}

El experimento se ubicó en la finca San Pablo, propiedad de CORBANA, en el cable seis sur, área conocida como $20 \mathrm{H}$.

\section{EXPERIMENTO \#1:}

\section{Duración:}

Inicio: Noviembre, 1996. Final: Agosto, 1998

Selección de las unidades experimentales:

Las unidades experimentales se seleccionaron al azar y se aleatorizaron el 6 de noviembre de 1996. De inmediato se procedió a identificarlas con un sistema triple: cintas plásticas, placas de aluminio y rasgado del código sobre el pseudotallo de las madres. Para facilitar la ubicación en el campo, las unidades pertenecientes a un mismo tratamiento se marcaron con el mismo color (en una sección del pseudotallo de las abuelas y en el extremo distal de estacas de bambú colocadas al frente de cada unidad). Al día siguiente, antes de asper- jar los tratamientos se hicieron mediciones del crecimiento sobre las unidades experimentales (Cuadro 1).

El 8 de noviembre de 1996 se aspetjó el primer ciclo con glifosato (tratamientos 1,2,3 y 4).

Diseño experimental y unidades experimentales:

Se usó el diseño irrestricto al azar, con 30 repeticiones para los tratamientos con glifosato y 52 repeticiones para el testigo de deshierba manual. El área experimental total fue de casi tres hectáreas del clon Gran Enano. Las repeticiones consistieron de unidades de producción seleccionadas en un estado fenológico similar (todas con abuela-madre-hijo, al inicio). Todas las abuelas mostraban racimos en diferente estado de desarrollo, que se anotó por medio del color de las cintas plásticas que se utilizan para el control de la cosecha.

\section{Tratamientos}

1. Aspersión directa (primer ciclo, 8 de noviembre, 1996) sobre hijo de tres a $25 \mathrm{~cm}$ de altura, con una solución al 2\% v/v de Ranger (glifosato 240 g/l).

2. Aspersión directa sobre el hijo, dos veces consecutivas con Ranger al 2\% v/v; la primera aspersión se realizó el mismo día que en tratamiento \#1, la segunda aspersión (21 de dicembre, 1996) sobre hijos que variaban entre 40 y $60 \mathrm{~cm}$ de altura, y todavía dependían de la madre (hojas con menos de tres $\mathrm{cm}$ de ancho).

3. Ciclos de aspersión consecutivos con solución de Ranger al $2 \%$ v/va intervalos de seis a siete semanas, hasta la cosecha. Los dos primeros ciclos en las mismas fechas que en el tratamientio 2. En cada ciclo, los hijos (eventualmente plantas adultas) se asperjaron de manera directa hasta una altura entre 50 y $60 \mathrm{~cm}$.

4. Similar que tratamiento \#3, pero se cubrieron los hijos con bolsas plásticas antes de cada aspersión, para asegurar que Ranger no se depositara sobre ellos. Además, se asperjaron los alrededores de la unidad experimental en un diámetro aproximado de seis metros, para simular una aspersión comercial.

5. Aspersión directa con solución de Ranger al 2\% v/v sobre pseudotallo de hijos, hasta una altura de 50 a $60 \mathrm{~cm}$. Durante la primera aspersión (8 de mayo 1997) esos hijos debían mostrar por lo menos una hoja con cinco o más cm de ancho en la región media y una altura mínima de $100 \mathrm{~cm}$. Esto se interpretó como inicio de independencia de la madre. 
Se continuó con ciclos cada seis o siete semanas hasta la cosecha con soluciones con el mismo herbicida y la misma concentración.

6. Control manual de malezas a intervalos aproximados de un mes.

Nota: En tratamientos con glifosato, el tiempo de aspersión sobre cada hijo fue de aproximadamente un segundo. La descarga promedio de la boquilla fue de $955 \mathrm{ml} / \mathrm{min}$.

Ciclos de aspersión: tiempo transcurrido entre una aspersión y la siguiente entre 6 y 7 semanas en este estudio.

\section{Equipo de aspersión}

Para asperjar el glifosato, se usó una bomba de espalda Carpi, provista con una boquilla TJ 8003; a una presión promedio de 32 psi.

\section{Toma de datos}

\section{A. En plantas madres:}

a1. Altura, Circunferencia a la base del pseudotallo al inicio del experimento y a un metro de altura en las siguientes evaluaciones. Fechas de evaluación: inicio del experimento, luego de la emergencia del racimo y a la cosecha.

B. En hijos productivos, horas antes de cada aspersión:

b1. Altura, medida desde la superficie del suelo hasta la unión foliar de la hoja más joven.

b2. Circunferencia del pseudotallo

b3. Ancho (en $\mathrm{cm})$ de la hoja más joven en la sección media.

b4. Número de hojas

C. estado de reproductivo:

c l. Fecha de cosecha

c2. Peso del racimo

c3. Número de manos

c4. Largo del dedo central de la segunda mano apical

c5. Calibración basal del mismo dedo

D. Otras variables:

d1. Altura y número de hojas en madres, el día de la floración de estas.

d2. Altura, número de hojas, ancho de la hoja más joven, circunferencia del seudotallo. Evaluadas en hijos, el día de la floración de las madres.

d3. Volumen radical, estimación del porcentaje de raíces activas y conteo de nematodos en las siguien tes fechas de muestreo:
Tratamiento 1:

45 días luego del primer ciclo con Ranger.

Tratamiento 2:

45 días después del segundo ciclo con Ranger.

Tratamiento 3:

45 días después del cuarto ciclo con Ranger.

Tratamiento 4:

igual que para tratamiento 3 .

Tratamiento 5:

en este tratamiento no se tomaron estas variables.

Tratamiento 6:

se evaluó cada vez que se muestreó alguno de los otros tratamientos.

Procedimiento para el muestreo de raíces:

Se utilizó el procedimiento para el estudio de ne matodos en plantas de banano (Araya et al, 1995). Las raíces que se localizaban en un pozo $(13 \mathrm{~cm}$ largo, 13 $\mathrm{cm}$ ancho y $30 \mathrm{~cm}$ hondo, para $5,07 \mathrm{~cm}^{3}$ de suelo) fueron extraídas. El pozo se hace al frente de la base de los hijos. Las muestras de raíces se colocaron en bolsas plásticas y se transportaron al laboratorio. Ahí, se lavaron para eliminarles el suelo y se separaron en dos grupos (raíces funcionales: blancas, amarillentas o café, pero carentes de tejido muerto; no funcionales: muertas). Después de dos horas de secado al ambiente, se pesó cada grlipo en balanza electrónica.

En las mismas muestras se realizó un estudio rutinario de nematodos, bajo el procedimiento descrito por Ayoub (1980) y Araya et al (1995).

\section{Análisis estadístico}

Con cada variable se llevó a cabo análisis de varianza.

\section{EXPERIMENTO \#2:}

Repetición del experimento \# 1, a partir de febrero de 1998, sobre las mismas cepas, para evaluar el uso acumulado del Ranger sobre cepas productivas de banano. Se estima que el experimento concluirá en Diciembre de 1999. Acá se incluyen datos de dos evaluaciones.

\section{RESULTADOS Y DISCUSIÓN}

El experimento se inició luego de seleccionar unidades experimentales tan homogéneas como fue posible (Cuadro 1).

Además de los datos antes del inicio, en este documento se incluyen variables de crecimiento del banano 
Cuadro 1. Circunferencia del pseudotallo y altura de madres e hijos, al inicio del experimento. Finca San Pablo, 28 Millas, Costa Rica. Noviembre 7, 1996.

\begin{tabular}{cccccc}
\hline \multirow{2}{*}{ Tratamiento } & \multicolumn{2}{c}{ Circunferencia $(\mathbf{c m})$} & & \multicolumn{2}{c}{ Altura $(\mathbf{c m})$} \\
\cline { 2 - 3 } \cline { 5 - 6 } & Madres & Hijos & & Madres & Hijos \\
\hline 1 & 66,9 & 12,5 & & 219,5 & 13,7 \\
2 & 63,8 & 11,4 & & 211,8 & 14,1 \\
3 & 68,4 & 11,2 & & 225,3 & 11,2 \\
4 & 66,1 & 11,5 & & 217,4 & 12,7 \\
5 & 65,9 & 11,8 & & 218,7 & 12,9 \\
6 & 68,3 & 11,3 & & 224,4 & 13,4 \\
CV & 13,1 & 27,4 & & 15,5 & 44,0 \\
\hline
\end{tabular}

No se encontraron diferencias entre tratamientos según análisis de varianza.

$\mathrm{CV}=$ coeficiente de variación $(\%)$

Cuadro 2. Variables de crecimiento en hijos, un día antes del quinto ciclo con el herbicida glifosato. Finca San Pablo, 28 Millas, Costa Rica. Mayo 8, 1997.

\begin{tabular}{ccccc}
\hline Tratamiento & $\begin{array}{c}\text { Altura } \\
(\mathbf{c m})\end{array}$ & $\begin{array}{c}\text { Circunferencia } \\
(\mathbf{c m})\end{array}$ & $\begin{array}{c}\text { Número de } \\
\text { hojas verdes }\end{array}$ & $\begin{array}{c}\text { Ancho de hoja } \\
\text { más joven }(\mathbf{c m})\end{array}$ \\
\hline 1 & 142,1 & 50,7 & 7,5 & 26,8 \\
2 & 129,8 & 49,7 & 7,3 & 24,3 \\
3 & 138,7 & 50,7 & 7,4 & 26,6 \\
4 & 142,2 & 51,6 & 7,5 & 25,3 \\
5 & 124,2 & 46,2 & 7,2 & 21,6 \\
6 & 142,4 & 51,9 & 7,4 & 26,6 \\
CV & 31,8 & 23,9 & 19,9 & 55,3 \\
\hline
\end{tabular}

No se encontraron diferencias entre tratamientos según análisis de varianza.

Cuadro 3. Variables de crecimiento en hijos bajo estudio (ahora adultos), un día antes del noveno ciclo con el herbicida glifosato. Finca San Pablo, 28 Millas. Octubre 22, 1997.

\begin{tabular}{|c|c|c|c|}
\hline Tratamiento & $\begin{array}{l}\text { Altura } \\
(\mathrm{cm})\end{array}$ & $\begin{array}{l}\text { Circunferencia del } \\
\text { pseudotallo }(\mathrm{cm})\end{array}$ & $\begin{array}{l}\text { Número de } \\
\text { hojas verdes }\end{array}$ \\
\hline $1 \quad(n=25)$ & 289,6 & 84,2 & 12,0 \\
\hline $2(\mathrm{n}=26)$ & 291,3 & 84,9 & 12,0 \\
\hline $3 \quad(n=26)$ & 285,4 & 83,9 & 12,4 \\
\hline $4 \quad(n=26)$ & 284,7 & 83,2 & 12,1 \\
\hline $5 \quad(\mathrm{n}=28)$ & 287,9 & 81,8 & 11,4 \\
\hline $6 \quad(n=41)$ & 286,1 & 84,5 & 12,0 \\
\hline $\mathrm{CV}$ & 12,9 & 12,7 & 13,6 \\
\hline
\end{tabular}

No se encontraron diferencias entre tratamientos según análisis de varianza. 
un día antes del quinto y noveno ciclos con glifosato (Cuadros 2 y 3), a efectos de evitar información reiterativa. No se observaron diferencias entre tratamientos en fechas de evaluación que precedieron a estas.

Los tratamientos tres y cuatro representan dos extremos. En las unidades de producción del tratamiento tres, cada seis a siete semanas se asperjaron los hijos productivos con la solución de Ranger al $2 \% \mathrm{v} / \mathrm{v}$ hasta una altura de 50 a $60 \mathrm{~cm}$, hijos con alturas inferiores a ese rango, recibieron el herbicida sobre la totalidad de la planta (hojuelas, pseudotallo); una vez que superaron esa altura, el producto cayó sobre el pseudotallo de estos. Estos hijos no se vieron afectados por el producto aun luego de ser asperjados ocho veces consecutivas, siempre y cuando se evitó el contacto con hojas fotosintéticamente activas (aquellas que aparecen cuando los hijos han superado el metro de altura). En el tratamiento cuatro se protegieron los hijos durante la aspersión, pero la misma se hizo en cada ocasión alrededor de la unidad, por lo que no afectó a los hijos desde el suelo, ante un uso consecutivo del producto.

Las aspersiones consecutivas con Ranger sobre hijos productivos no afectaron la cantidad ni la calidad de la fruta en las madres de esos hijos (Cuadros 4 y 5) y tampoco en los hijos asperjados (Cuadros 6 y 7).

Otro aspecto importante en la producción bananera es el crecimiento sincronizado entre madres e hijos. Esto no se vio afectado por ninguno de los tratamientos, en relación con el testigo deshierbado (Cuadro 8).

No se detectaron diferencias entre tratamientos en el peso total de raíces, ni en en el peso de raíces funcionales o no funcionales. Por otra parte, las poblaciones del nematodo Radopholus similis tampoco mostraron diferencias (Cuadro 9).

Cuadro 4. Variables de rendimiento en madres de hijos asperjados con el herbicida glifosato.

\begin{tabular}{cccc}
\hline Tratamiento & $\begin{array}{c}\text { Peso del } \\
\text { racimo }(\mathbf{k g})\end{array}$ & $\begin{array}{c}\text { Peso del pinzote } \\
(\mathbf{k g})\end{array}$ & $\begin{array}{c}\text { Número } \\
\text { de manos }\end{array}$ \\
\hline $1(\mathrm{n}=27)$ & 35,3 & 3,3 & 8,4 \\
$2(\mathrm{n}=26)$ & 34,2 & 3,1 & 8,5 \\
$3(\mathrm{n}=26)$ & 35,1 & 3,4 & 8,5 \\
$4(\mathrm{n}=25)$ & 34,2 & 3,3 & 8,0 \\
$5(\mathrm{n}=22)$ & 36,8 & 3,4 & 8,6 \\
$6(\mathrm{n}=44)$ & 36,7 & 3,4 & 8,6 \\
CV & 19,7 & 24,9 & 15,2 \\
\hline
\end{tabular}

No se encontraron diferencias entre tratamientos según análisis de varianza.

Cuadro 5. Variables de calidad de fruta en madres de hijos asperjados con el herbicida glifosato.

\begin{tabular}{cccccc}
\hline \multirow{2}{*}{ Tratamiento } & \multicolumn{2}{c}{ Calibración } & & \multicolumn{2}{c}{ Longitud } \\
\cline { 2 - 3 } \cline { 5 - 6 } & SEG & ULT & & SEG & ULT \\
\hline $1(n=27)$ & 44,6 & 40,7 & & 24,6 & 20,7 \\
$2(n=26)$ & 44,6 & 40,9 & & 24,8 & 20,9 \\
$3(n=26)$ & 45,2 & 41,1 & & 24,9 & 20,8 \\
$4(n=25)$ & 44,8 & 41,0 & & 24,8 & 21,1 \\
$5(n=22)$ & 45,3 & 41,1 & & 24,8 & 20,9 \\
$6(n=44)$ & 45,0 & 41,1 & & 24,8 & 21,0 \\
CV & 2,4 & 3,6 & & 5,5 & 6,4 \\
\hline \multicolumn{3}{l}{ SEG = segunda mano } \\
No se encontraron diferencias entre tratamientos según análisis de varianza.
\end{tabular}


Cuadro 6. Variables de rendimiento en hijos de banano asperjados con el herbicida glifosato.

\begin{tabular}{cccc}
\hline Tratamiento & $\begin{array}{c}\text { Peso del } \\
\text { racimo }(\mathbf{k g})\end{array}$ & $\begin{array}{c}\text { Peso del pinzote } \\
(\mathbf{k g})\end{array}$ & $\begin{array}{c}\text { Número } \\
\text { de manos }\end{array}$ \\
\hline $1(\mathrm{n}=26)$ & 34,6 & 3,2 & 8,4 \\
$2(\mathrm{n}=28)$ & 33,7 & 3,0 & 8,1 \\
$3(\mathrm{n}=27)$ & 35,6 & 3,1 & 8,6 \\
$4(\mathrm{n}=28)$ & 35,2 & 3,3 & 8,4 \\
$5(\mathrm{n}=25)$ & 35,3 & 3,3 & 8,2 \\
$6(\mathrm{n}=46)$ & 35,6 & 3,2 & 8,2 \\
\hline $\mathrm{CV}$ & 18,5 & 23,3 & 13,5 \\
\hline
\end{tabular}

No se encontraron diferencias entre tratamientos según análisis de varianza.

Cuadro 7. Variables de calidad de fruta en hijos de banano asperjados con el herbicida glifosato.

\begin{tabular}{llllll}
\hline \multirow{2}{*}{ Tratamiento } & \multicolumn{2}{c}{ Calibración } & & \multicolumn{2}{c}{ Longitud } \\
\cline { 2 - 3 } \cline { 5 - 6 } & SEG & ULT & & SEG & ULT \\
\hline $1(n=26)$ & 45,1 & 41,6 & & 24,1 & 20,3 \\
$2(n=28)$ & 45,3 & 41,5 & & 24,6 & 20,4 \\
$3(n=27)$ & 45,4 & 41,6 & & 24,6 & 20,9 \\
$4(n=28)$ & 45,4 & 41,4 & & 24,6 & 20,6 \\
$5(n=25)$ & 45,3 & 41,8 & & 24,9 & 21,1 \\
$6(n=46)$ & 45,8 & 42,2 & & 24,7 & 21,0 \\
CV & 2,8 & 3,9 & & 5,3 & 6,9 \\
\hline
\end{tabular}

$\mathrm{SEG}=$ segunda mano $\quad \mathrm{ULT}=$ última mano

No se encontraron diferencias entre tratamientos según análisis de varianza.

Cuadro 8. Variables de crecimiento de madres e hijos de banano asperjados con glifosato, el día de la floración de las madres.

\begin{tabular}{|c|c|c|c|c|c|c|}
\hline \multirow[t]{2}{*}{ Tratamiento } & \multicolumn{2}{|c|}{ Madres a floración } & \multirow[b]{2}{*}{$\begin{array}{c}\text { Altura } \\
(\mathbf{c m})\end{array}$} & \multicolumn{3}{|c|}{ Hijos asperjados } \\
\hline & $\begin{array}{c}\text { Altura } \\
\text { (cm) }\end{array}$ & $\begin{array}{l}\text { Número } \\
\text { de hojas* }\end{array}$ & & $\begin{array}{l}\text { Circun } \\
(\mathrm{cm})\end{array}$ & $\begin{array}{l}\text { \# de } \\
\text { hojas }\end{array}$ & $\begin{array}{l}\text { Ancho de hoja } \\
\text { más joven }(\mathrm{cm})\end{array}$ \\
\hline 1 & 324,2 & 14 & 152,7 & 53,8 & 7,9 & 25,7 \\
\hline 2 & 330,5 & 14 & 142,3 & 52,3 & 7,5 & 20,5 \\
\hline 3 & 322,3 & 14 & 147,7 & 52,4 & 7,6 & 23,4 \\
\hline 4 & 328,5 & 14 & 150,7 & 53,2 & 7,6 & 23,7 \\
\hline 5 & 323,9 & 14 & 142,4 & 49,9 & 7,4 & 20,6 \\
\hline 6 & 323,9 & 14 & 150,8 & 53,5 & 7,6 & 24,5 \\
\hline $\mathrm{CV}$ & 8,5 & 7,8 & 24,5 & 20,4 & 19,2 & 63,2 \\
\hline
\end{tabular}

No se encontraron diferencias entre tratamientos según análisis de varianza.

$*$ Datos redondeados $( \pm 0,25)$. 
Cuadro 9. Variables de las raíces de los hijos de banano bajo estudio.

\begin{tabular}{lccc}
\hline Variable & Tratamiento 3 & Tratamiento 4 & $\begin{array}{c}\text { Tratamiento 6 } \\
\text { (testigo) }\end{array}$ \\
\hline Raíz total (g) & 36 & 43,8 & 32,2 \\
Raíz funcional (g) & 23,8 & 38,2 & 28,0 \\
Raíz no funcional (g) & 8,4 & 5,6 & 4,2 \\
Radopholus & 11.456 & 18.880 & 18.543 \\
similis/100 g raíz & & & \\
Altura del hijo (cm) & 253 & 233 & 245 \\
\hline
\end{tabular}

No se encontraron diferencias entre tratamientos según análisis de varianza.

T3 = 45 días después de la sexta aplicación con Glifosato (Ranger $240 \mathrm{~g} / \mathrm{l}$ )

$\mathrm{T} 4$ = similar a T3, pero se evitó contacto directo de Glifosato (Ranger $240 \mathrm{~g} / \mathrm{l}$ ) con hijos

T6 = 1 chapia c/mes; muestreado el mismo día que T3 y T4

El muestreo se hizo al frente del hijo con palín, $5070 \mathrm{~cm}^{3}$ de suelo/planta, 5 plantas-

/tratamiento

\section{EXPERIMENTO \# 2:}

En este documento se presentan datos del inicio del estudio (Cuadro 10) y los últimos con que se cuenta procesados, poco antes del quinto ciclo con el herbicida Ranger (Cuadro 11), para evitar información reiterativa.

Se siguió la misma metodología que para el experimento \#1. Aquellas unidades de producción que no presentaban hijos productivos al inicio de este estudio se eliminaron, pues no habrían recibido el herbicida en uno o varios ciclos, dependiendo del tratamiento. Como era de esperar, los hijos ya emergidos que se evaluaron en este segundo experimento, mostraron una alta variación en tamaño poco antes del inicio (ver coeficientes de variación en Cuadro 10), en vista de que en esta ocasión no se podían seleccionar por ese criterio. En todo caso, los coeficientes de variación bajaron a niveles co- mo los del primer experimento, luego de iniciados los tratamientos, para la evaluación que se presenta (Cuadro 11).

Se contó para este segundo experimento con más de 20 repeticiones para los tratamientos con glifosato y con 36 repeticiones para el testigo deshierbado. El atraso en la emisión de hijos productivos en algunas unidades es propio de la fisiología del banano y no se relaciona con el uso del herbicida, pues fue similar en todos los tratamientos y es un comportamiento conocido por los productores.

\section{CONCLUSIONES}

1. El uso en ciclos de aspersión consecutivos del herbicida glifosato sobre hijos productivos de banano,

Cuadro 10. Variables de crecimiento en hijos productivos de banano, al inicio del experimento \# 2. Finca San Pablo, 28 Millas, Costa Rica. Febrero 27, 1998.

\begin{tabular}{lccc}
\hline Tratamiento & Altura $(\mathbf{c m})$ & $\begin{array}{c}\text { Circunferencia }(\mathbf{c m}) \\
\text { base pseudotallo }\end{array}$ & $\begin{array}{c}\text { \# de hojuelas } \\
\text { (no fotosintéticas) }\end{array}$ \\
\hline $1(\mathrm{n}=22)$ & 24,9 & 14,2 & 2,3 \\
$2(\mathrm{n}=24)$ & 23,6 & 13,9 & 2,2 \\
$3(\mathrm{n}=23)$ & 22,8 & 12,5 & 2,1 \\
$4(\mathrm{n}=22)$ & 20,7 & 11,8 & 2,0 \\
$5(\mathrm{n}=21)$ & 20,1 & 12,0 & 2,0 \\
$6(\mathrm{n}=36)$ & 21,4 & 12,9 & 1,9 \\
CV & 102,3 & 62,0 & 85,4 \\
\hline
\end{tabular}

No se encontraron diferencias entre tratamientos según análisis de varianza. 